\title{
DifFuse OPTICAL TOMOgRAPHY BASED ON A WKB APPROXIMATION
}

\author{
Fanan Zeng, Gang Zhang, Feng Liu, and R. R. Alfano \\ The City College and the Graduate School of the City University of New York, Institute for Ultrafast \\ Spectroscopy and Lasers, New York State Center of Advanced Technology for Ultrafast Photonic \\ Materials and Applications, Department of Physics and Department of Electrical Engineering, New \\ York, New York 10031 \\ (Paper JBO-100 received June 26, 1996; revised manuscript received June 13, 1997; accepted for publication July 29, 1997.)
}

\begin{abstract}
An image reconstruction algorithm based on a Wentzel-Kramers-Brillouin (WKB) approximation in the frequency domain in terms of path integration is tested with both simulated and experimental data. The algorithm can be used with full frequency domain data (amplitude modulation and phase shift), or partial data (amplitude or phase shift). This algorithm reconstructs an image of photon density wave propagation factor $\kappa$ without knowing the optical parameters of the background medium. Reconstruction was performed for data sets of different modulation frequencies and different detector geometries. The reconstruction time on a SUN-SPARC 5 station was about $5 \mathrm{~min}$ for images with a $128 \times 128$ pixel size. () 1997 Society of Photo-Optical Instrumentation Engineers. [S1083-3668(97)01104-0]
\end{abstract}

Keywords NIR image reconstruction algorithm; WKB approximation; multiple scattering; random media.

\section{INTRODUCTION}

Researchers are still hindered by the inherent difficulties in transforming near-infrared (NIR) transmission and reflection data from highly scattering random media to form images of human breast and brain for clinical use. The central problem stems from the fact that a major portion of the photon transport in human tissue in the NIR or visible wavelength range is diffusive due to strong multiple light scattering. Unlike X-ray computed tomography (CT), which deals with photons that travel along the straight line of sight between source and detector (ballistic photons), owing to multiple scattering in the region, NIR photons travel from source to detector within a rather large banana-shaped region inside the tissue. ${ }^{1}$ From a set of diffuse light intensities measured on the tissue surface, it is much more difficult to generate a sharp map or image of the structures inside the tissue using diffusion theory.

The possibility of using NIR light to reconstruct the image of objects that have various optical parameters hidden in highly scattering media has recently being investigated by many researchers with the aim of using it for imaging the human body. ${ }^{2-12}$ In order to calculate the so-called weight function, almost all these image reconstruction methods need to know the optical parameters of a reference medium, which may be difficult for practical use. Recently, Feng, Zhao, and Zeng ${ }^{13}$ and Feng, Zeng, and

Address all correspondence to R. R. Alfano. E-mail: alfano@sci.ccny.cuny.edu
Zhao $^{14}$ developed a novel inverse algorithm to address some of these problems. The algorithm is based on a Wentzel-Kramers-Brillouin (WKB) approximation of diffusion theory.

In this paper, the results of image reconstruction using both simulated and experimental data in a frequency domain are presented. Reconstruction using data of different modulation frequencies and different source detector geometries is investigated. The forward solutions of the diffusion equation based on the WKB approximation in the path integral form, the inverse algorithm, and computation procedures are described in Sec. 2. In Sec. 3, the results of image reconstruction for infinite and finite random media as well as experimental results in an infinite medium are presented. Section 4 contains the discussion and conclusions.

\section{WKB APPROXIMATION AND IMAGE RECONSTRUCTION ALGORITHM FOR HIGHLY SCATTERING RANDOM MEDIA}

NIR photon migration in tissue can be described by the diffusion equation when the length scale under consideration $L$ is large compared with the transport mean free path $l^{*}=1 / \mu_{s}^{\prime}\left(\mu_{s}^{\prime}\right.$ being the transport scattering coefficient), $L / l^{*} \gg 1$ :

$$
\frac{1}{c} \frac{\partial \phi}{\partial t}=\nabla(D \nabla \phi)-\mu_{a}(\mathbf{x}) \phi+\eta(\mathbf{x}, t)
$$


where $\phi(\mathbf{x}, t)$ is the diffuse photon fluence rate, $c$ is the speed of light in tissue, $\mu_{a}(\mathbf{x})$ is the positiondependent absorption coefficient, $\eta(\mathbf{x}, t)$ represents an isotropic light source, and $D=\left[1 / 3\left(\mu_{a}+\mu_{s}^{\prime}\right)\right]$ is the diffusion constant.

In the case of an instantaneous pointlike light source, $\eta(\mathbf{x}, t)=\delta(\mathbf{r}-\mathbf{a}) \delta(t)$, the solution of Eq. (1) is the time domain Green's function. For an amplitude-modulated point source, $\eta(\mathbf{x}, t)=\delta(\mathbf{r}$ $-\mathbf{a}) \exp (-i \omega t)$, the solution of Eq. (1) is the frequency domain Green's function.

The important inverse problem is how to reconstruct the position, shape, size, and optical parameters, such as $D$ and $\mu_{a}$, of one or a few inhomogeneities in a random medium from measurement signals $\phi(\mathbf{r})$ made on the boundary or inside the medium.

\subsection{FORWARD ALGORITHM BASED ON THE WKB APPROXIMATION}

The diffusion equation (1) can be described by path integration following the original work of Feynman; ${ }^{15}$ the forward problem of diffusion Eq. (1) can be obtained in both time and frequency domains. The details of the path integral formula are presented in the appendix. The forward solution in the frequency domain for diffuse photons propagating from source point $\mathbf{a}$ to detection point $\mathbf{b}$ is given by:

$$
\phi(\mathbf{b}, \mathbf{a} ; \omega)=\left(\frac{1}{2 \pi}\right)^{(d-1 / 2)} \sqrt{\tilde{D}} \exp [-W(\mathbf{b}, \mathbf{a} ; \omega)],
$$

where $d=3$ is the spatial dimension and $W(\mathbf{b}, \mathbf{a} ; \omega)$ is given by:

$$
W(\mathbf{b}, \mathbf{a} ; \omega)=\int_{\mathbf{a}}^{\mathbf{b}} \kappa(\mathbf{x}, \omega) d \mathbf{l},
$$

where $\kappa(\mathbf{x}, \omega)=\left(\mu_{a} / D-i \omega / D c\right)^{1 / 2}$ is the photon density wave propagation vector, $d \mathbf{l}$ is a path element, and the integration is performed over the most probable diffusing path (MPDP). For a uniform scattering medium, the MPDP is a straight line between source and detector [see Eq. (21)].

$\tilde{D}$ is the path's density prefactor and is given by:

$$
\begin{aligned}
\tilde{D} & =\operatorname{det}\left(\frac{\partial^{2} \bar{S}\left(\mathbf{b}, \mathbf{a} ; t_{0}\right)}{\partial \mathbf{b} \partial \mathbf{a}}\right) / \frac{\partial^{2} \bar{S}\left(\mathbf{b}, \mathbf{a} ; t_{0}\right)}{\partial t_{0}^{2}} \\
& =\operatorname{det}\left[\begin{array}{cc}
\frac{\partial^{2} W}{\partial \mathbf{b} \partial \mathbf{a}} & i \frac{\partial^{2} W}{\partial \mathbf{b} \partial \omega} \\
i \frac{\partial^{2} W}{\partial \mathbf{a} \partial \omega} & -\frac{\partial^{2} W}{\partial \omega^{2}}
\end{array}\right] .
\end{aligned}
$$

\subsection{INVERSE ALGORITHM BASED ON THE WKB APPROXIMATION}

The inverse problem, determining $\mu_{a}(\mathbf{x})$ or $\mu_{s}^{\prime}(\mathbf{x})$, or both from measurements on the boundary of an inhomogeneous tissue system such as the human brain or breast, is a difficult problem. The difficulty lies in the fact that in order to relate $\mu_{a}(\mathbf{x})$, or $\mu_{s}^{\prime}(\mathbf{x})$, or both inside the tissue to the measured signals $\phi(\mathbf{b}, \mathbf{a}, \omega)$ and $\phi_{0}(\mathbf{b}, \mathbf{a}, \omega)$ (corresponding to with and without an object) at some tissue surface position $\mathbf{b}$, given the source location a on another point of the tissue surface, one needs to know the most probable diffusing path $\overline{\mathbf{x}}(\tau)$ in a time domain, or $\overline{\mathbf{x}}(l)$ in a frequency domain (see the appendix). The exact shape of the MPDP itself is determined by the distribution of $\mu_{a}(\mathbf{x})$ and $\mu_{s}^{\prime}(\mathbf{x})$, which are unknown. At present, there does not exist a general solution to this fundamental problem. However, when inhomogeneity can be regarded as small, using the MPDP for a homogeneous medium (straight-line path) is a good approximation and one can seek a perturbation solution. Equation (3) can be approximated as

$$
W(\mathbf{b}, \mathbf{a} ; \omega)=\int_{\mathbf{a}}^{\mathbf{b}} \kappa(\mathbf{x}, \omega) d \mathbf{l} \approx \kappa_{0}|\mathbf{b}-\mathbf{a}|+\int_{\mathbf{a}}^{\mathbf{b}} \delta \kappa(\mathbf{x}, \omega) d \mathbf{l},
$$

where $\delta \kappa(\mathbf{x}, \omega)=\kappa(\mathbf{x}, \omega)-\kappa_{0}(\mathbf{x}, \omega)$ is the change of complex propagation factor $\kappa$ due to inhomogeneity.

Substituting Eq. (5) in Eq. (2) and combining all factors in the previous exponent of Eq. (2), with the background signal defined as $\phi_{0}(\mathbf{b}, \mathbf{a}, \omega)$, the corresponding equation for Eq. (2) may be approximated as

$$
\phi(\mathbf{b}, \mathbf{a}, \omega) \approx \phi_{0}(\mathbf{b}, \mathbf{a}, \omega) \exp \left[-\int_{\mathbf{a}}^{\mathbf{b}} \delta \kappa(\mathbf{x}, \omega) d l\right],
$$

where $\phi(\mathbf{b}, \mathbf{a}, \omega)$ and $\phi_{0}(\mathbf{b}, \mathbf{a}, \omega)$ are signals with and without an object, respectively. Equation (6) is a complex and can be written as real and imaginary parts:

$$
\ln \left(\left|\phi / \phi_{0}\right|\right)=\operatorname{real}\left[\ln \left(\phi / \phi_{0}\right)\right]=-\int_{\mathbf{a}}^{\mathbf{b}} \delta \kappa_{r}(\mathbf{x}, \omega) d l,
$$

and

$$
\Phi=\operatorname{imag}\left[\ln \left(\phi / \phi_{0}\right)\right]=-\int_{\mathbf{a}}^{\mathbf{b}} \delta \kappa_{i}(\mathbf{x}, \omega) d l .
$$

where $\Phi$ is the phase shift and $\delta \kappa_{r}(\mathbf{x}, \omega)$ and $\delta \kappa_{i}(\mathbf{x}, \omega)$ are the real and imaginary parts of $\delta \kappa(\mathbf{x}, \omega)$ to be solved, respectively. Equations (7) and (8) show a direct relation between the change in measured intensity signal and the changes in propaga- 
tion factor of photon density wave $\delta \kappa_{r}$ and $\delta \kappa_{i}$. From Eq. (32), the real part and imaginary parts of $\delta \kappa$ are:

$$
\begin{aligned}
\delta \kappa_{r}= & \frac{1}{\sqrt{2 D}}\left(\sqrt{\mu_{a}^{2}+\omega^{2} / c^{2}}+\mu_{a}\right)^{1 / 2} \\
& -\frac{1}{\sqrt{2 D_{0}}}\left(\sqrt{\mu_{a 0}^{2}+\omega^{2} / c^{2}}+\mu_{a 0}\right)^{1 / 2}
\end{aligned}
$$

and

$$
\begin{aligned}
\delta \kappa_{i}= & \frac{1}{\sqrt{2 D}}\left(\sqrt{\mu_{a}^{2}+\omega^{2} / c^{2}}-\mu_{a}\right)^{1 / 2} \\
& -\frac{1}{\sqrt{2 D_{0}}}\left(\sqrt{\mu_{a 0}^{2}+\omega^{2} / c^{2}}-\mu_{a 0}\right)^{1 / 2}
\end{aligned}
$$

where $\mu_{a 0}$ and $D_{0}$ are the absorption coefficient and diffusion coefficient of the background medium and $\mu_{a}$ and $D$ are the absorption coefficient and diffusion coefficient of the inhomogeneous medium. Equations (9) and (10) show that $\delta \kappa_{r}$ and $\delta \kappa_{i}$ are related to both the change in $\mu_{a}$ and $\mu_{s}^{\prime}$.

To relate the signal change to the changes of absorption coefficient $\mu_{a}$ and scattering coefficient $\mu_{s}^{\prime}$, we can expand Eq. (6) in terms of the changes of $\delta \mu_{a}$ and $\delta \mu_{s}^{\prime}$. After expanding to the first order of variation, the signal is related to the change in optical parameters $\delta \mu_{a}$ and $\delta \mu_{s}^{\prime}$ as:

$$
\begin{aligned}
\phi(\mathbf{b}, \mathbf{a}, \omega)= & \phi_{0}(\mathbf{b}, \mathbf{a}, \omega) \exp \left[-\frac{\kappa_{0}^{*}}{2 \sqrt{\mu_{a 0}^{2}+(\omega / c)^{2}}}\right. \\
& \left.\times \int_{\mathbf{a}}^{\mathbf{b}} \delta \mu_{a}(\mathbf{x}) d l+\frac{\kappa_{0}}{2 D_{0}} \int_{\mathbf{a}}^{\mathbf{b}} \delta D(\mathbf{x}) d l\right] .
\end{aligned}
$$

Equations (7), (8), and (11) are forward equations relating the change in optical properties to the change intensity signal and are key for the WKB image reconstruction algorithm. Equation (11) can be used to reconstruct the spatial maps of optical parameters $\delta \mu_{a}$ and $\delta \mu_{s}^{\prime}$, while Eqs. (7) and (8) can be used to reconstruct the spatial maps of wave vectors $\delta \kappa_{r}$ and $\delta \kappa_{i}$. A comparison of Eqs. (7), (8), and (11) shows that for Eqs. (7) and (8) it is not necessary to know the optical parameters of the background media, and that computer memory is required for half of Eq. (11). Therefore, Eqs. (7) and (8) are easier than Eq. (11) for practical use. We will employ Eqs. (7) and (8) to reconstruct the image in the following paragraphs.

For a uniform change in absorption coefficient, the WKB approximation is exact, while for a uniform change in scattering coefficient $\left(D_{0} \rightarrow D\right)$, WKB is exact to a factor of $D / D_{0}$.

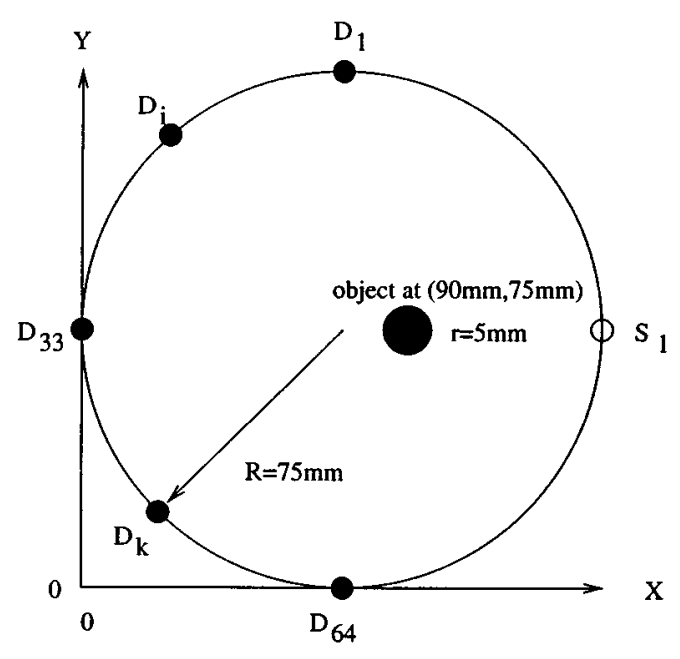

Fig. 1 Schematic of fan beam geometry for simulated data in an infinite medium.

To reconstruct an image to form a contour map of objects, the reconstruction region is first partitioned into a lattice, with each cell representing a pixel (voxel). Thus, Eqs. (7) and (8) are discretized on a lattice:

$$
\sum_{j} \Delta l_{i j} \delta \kappa_{j}=\left(\ln \frac{\phi_{0}}{\phi}\right)_{i} \equiv p_{i},
$$

where $\delta \kappa_{j}$ represents $\delta \kappa_{r}$ or $\delta \kappa_{i}$ to be solved for the $j$ 'th pixel, $\Delta l_{i j}$ corresponds to that portion of the $i^{\prime}$ th straight line $\mathbf{a b}$ which falls into the $j^{\prime}$ th pixel, and $p_{i}$ represents the natural logarithm of the ratio (without/with) of the $i^{\prime}$ th pair signal of source and detector. The linear equations resulting from Eq. (12) can be solved in several ways. We employed the standard simultaneous algebraic reconstruction technique (SART), ${ }^{16}$ i.e.,

$$
\delta \kappa_{j}^{(k)}=\delta \kappa_{j}^{(k-1)}+\frac{\Sigma_{i}\left[l_{i j}\left[p_{i}-\Sigma_{j} l_{i j} \delta \kappa_{j}^{(k-1)}\right] / \Sigma_{j} l_{i j}\right]}{\Sigma_{i} l_{i j}},
$$

where $i$ is the $i$ 'th pair of source and detector, $j$ is the $j^{\prime}$ th pixel, and $k$ is the $k^{\prime}$ th iteration.

\section{Testing THE WKB IMAge RECONSTRUCTION AlgORITHM}

In this section we present image reconstruction using WKB imaging algorithms with both simulated and experimental data.

\subsection{IMAGE RECONSTRUCTION IN AN INFINITE MEDIUM USING SIMULATED DATA}

In order to simulate a tumor in the human brain or breast in an infinite medium, a model system (see Figure 1) was used that consisted of a spherical object with a diameter of $10 \mathrm{~mm}$, off center at $(90 \mathrm{~mm}$, 


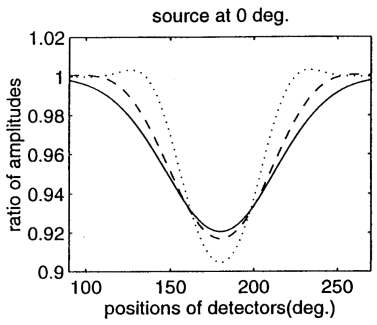

(a)

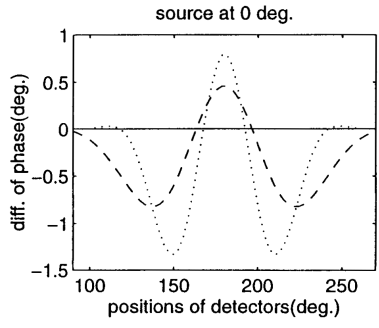

(b)
Fig. 2 Relationship of ratio $\left(\ln \left|\phi / \phi_{0}\right|\right)$ of the amplitudes and the phase shift difference (with object-without one) for various modulation frequencies where the source is at the angle $0 \mathrm{deg}$, and the 64 detectors are uniformly distributed on the half circle between $[\pi / 2,3 \pi / 2]$. Solid, dashed, and dotted lines correspond to results for modulation frequencies ( $f$ ) of 0,200 , and $1000 \mathrm{MHz}$, respectively.

$75 \mathrm{~mm}, 0)$ and hidden inside the reconstruction region of a cylinder with a diameter of $150 \mathrm{~mm}$, centered at $(75 \mathrm{~mm}, 75 \mathrm{~mm}, 0)$ in an infinite medium.

The optical parameters for the background medium were chosen to correspond realistically to those of white matter in the human brain ${ }^{17-20}: l_{a 0}$ $=333 \mathrm{~mm} \quad\left(\mu_{a 0}=0.003 \mathrm{~mm}^{-1}\right)$, and $l_{t 0}=0.67 \mathrm{~mm}$ $\left(\mu_{s 0}^{\prime}=1.6 \mathrm{~mm}^{-1}\right)$. The absorbing spherical object was assumed to have optical parameters corresponding to those of a realistic hematoma, with a higher absorption $\tilde{l}_{a 1}=33 \mathrm{~mm}\left(\tilde{\mu}_{a 1}=0.03 \mathrm{~mm}^{-1}\right)$, and the same transport length as the background $\tilde{l}_{t 1}=0.67 \mathrm{~mm} \quad\left(\tilde{\mu}_{s 1}^{\prime}=1.6 \mathrm{~mm}^{-1}\right)$. The mock data "measured" at different modulation frequencies were computed at the various detection positions on $z=0$ plane by using an analytic solution by $\mathrm{O}^{\prime}$ Leary et al. ${ }^{4}$ For a source position, the photon fluence rates were computed at 64 equally spaced detector positions on the half circle opposite the source. For example, if the source was at $\theta=0 \mathrm{deg}$ (i.e., coordinates at $150 \mathrm{~mm}, 75 \mathrm{~mm}, 0$ ), then detectors were placed on the half circle uniformly within the angular range $[\pi / 2,3 \pi / 2]$. The source and detector pairs were rotated around the circle in 128 steps, providing data for 128 viewing angles. The total number of data points input for inverse reconstruction was $128 \times 64=8192$.

Figure 2 shows the relationship of the ratio (with object/without one) of the amplitudes and the phase-shift difference (with object-without one) for different modulation frequencies, where the source was at the angle 0 deg. Figures $2(a)$ and 2(b) display the ratio of amplitude modulation versus positions of detectors and the phase-shift difference versus the positions of detectors, respectively. Changes in amplitude and phase are small because the object is very small (the ratio of the cross section of the sampling area and the object is $1 / 225 \approx 0.5 \%$ ).

The AC amplitude in most detectors decreases as the absorbing object is inserted into the medium. The maximum reduction occurs when the detector is placed in the line of sight with the source and the
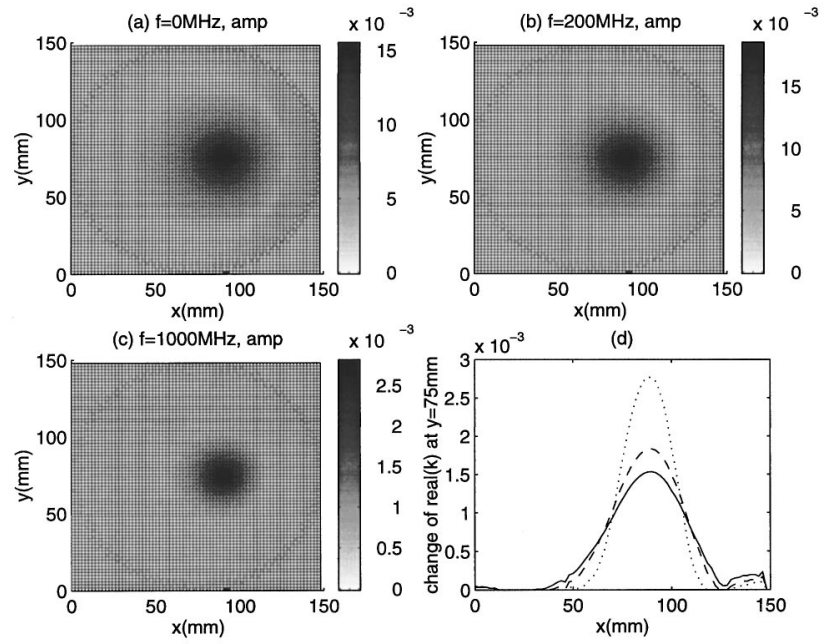

Fig. 32 2-D test of $\delta \kappa_{r}(\mathbf{x})$ image reconstruction of $128 \times 128$ pixels. Solid, dashed, and dotted lines in (d) correspond to the modulation frequencies of 0,200 , and $1000 \mathrm{MHz}$.

object. The decrease in amplitude indicates that the object has a higher scattering or absorption coefficient, or a higher $\kappa$ value.

The change in phase is interesting. The phase shift shows a decrease in phase for off-line-of-sight detectors but an increase in the line-of-sight detectors. This is an indication that the object might be an absorber. For off-line detectors, photons traveling through longer paths (off a straight sourcedetector line) and absorbed by the object result in a decrease in phase shift, while for on-line detectors, earlier arriving photons traveling through the object are absorbed, resulting in an increase in phase shift.

Figure 3 shows a test image reconstructed from simulated data for various modulation frequencies by using amplitudes of signals and Eq. (7). Figures $3(\mathrm{a}), 3(\mathrm{~b})$, and 3(c) are gray-scale representations of the reconstructed images of the real part of a wave vector $\delta \kappa_{r}(\mathbf{x}, f)$ for modulation frequencies of 0 , 200, and $1000 \mathrm{MHz}$, respectively. Figure 3(d) plots $\delta \kappa_{r}(\mathbf{x}, f)$ against spatial coordinates $x$ at $y$ $=75 \mathrm{~mm}$, i.e., the maximum slice plane of $\delta \kappa_{r}(\mathbf{x}, f)$. The central position and the shape of the reconstructed object are correct for all three modulation frequencies of 0,200 , and $1000 \mathrm{MHz}$. The maximum values for $\left.\delta \kappa_{r}\right|_{\max }$ and full-width-of-half maximum (FWHM) of the reconstructed object for modulation frequencies $f=0,200$, and $1000 \mathrm{MHz}$ are given in Table 1 . These values show that the reconstruction is larger than the real object (10 $\mathrm{mm}$ ). The size of the reconstructed object becomes smaller when the modulation frequency is increased.

Figure 4 shows a test image reconstructed from simulated data for modulation frequencies at 200 and $1000 \mathrm{MHz}$ by using imaginary parts of the signals $\left[\operatorname{imag}\left[\ln \left(\phi / \phi_{0}\right)\right]\right.$ and Eq. (8)]. Figures 4(a) and 4(c) are gray-scale representations of the recon- 
Table 1 Effect of modulation frequency in infinite media.

\begin{tabular}{rccccc}
\hline$f(\mathrm{MHz})$ & $\left.\delta \kappa_{r}\right|_{\max }\left(\mathrm{mm}^{-1}\right)$ & $\left.\delta \kappa_{i}\right|_{\max }\left(\mathrm{mm}^{-1}\right)$ & $H W_{r}(\mathrm{~mm})$ & $H W_{i}(\mathrm{~mm})$ & Object size $(\mathrm{mm})$ \\
\hline 0 & 0.00154 & $\mathrm{~N} / \mathrm{A}^{\mathrm{a}}$ & 42 & $\mathrm{~N} / \mathrm{A}$ & 10 \\
200 & 0.00184 & 0.00062 & 40 & 24 & 10 \\
1000 & 0.00277 & 0.00134 & 32 & 14 & 10 \\
\hline
\end{tabular}

a N/A:not applicable.

structed images $\delta \kappa_{i}(\mathbf{x}, f)$ and correspond to $f=200$ and $1000 \mathrm{MHz}$, respectively. Figures 4(b) and 4(d) show a plot of $\delta \kappa_{i}(\mathbf{x}, f)$ against spatial coordinates $x$ at $y=75 \mathrm{~mm}$, i.e., the maximum slice plane of $\delta \kappa_{i}$, corresponding to $f=200$ and $1000 \mathrm{MHz}$, respectively. These results are interesting. The central position of the reconstructed object is correct. The FWHM of the reconstructed object using the imaginary part is smaller than the results obtained from the real part of the data. The maximum values and FWHM are given in Table 1.

These values show that when $f=1000 \mathrm{MHz}$, the size of the image is almost the same as that of the real object $(10 \mathrm{~mm})$. Using imaginary reconstruction at the higher modulation frequency, the central position of the object and the approximation of its size can be obtained.

This is physically associated with the fact that as $\omega$ increases, the effective absorption length $L_{a}$ $\equiv 1 / \kappa^{\prime}$ decreases

$$
\left(\kappa^{\prime}=\operatorname{real}(\kappa)=\frac{1}{\sqrt{2 D_{0}}}\left[\sqrt{\mu_{a}^{2}+(\omega / c)^{2}}+\mu_{a}\right]^{1 / 2}\right),
$$

leading to a shrinkage in the width of the "banana" region connecting the source and detector in which photon diffusion paths are concentrated. This
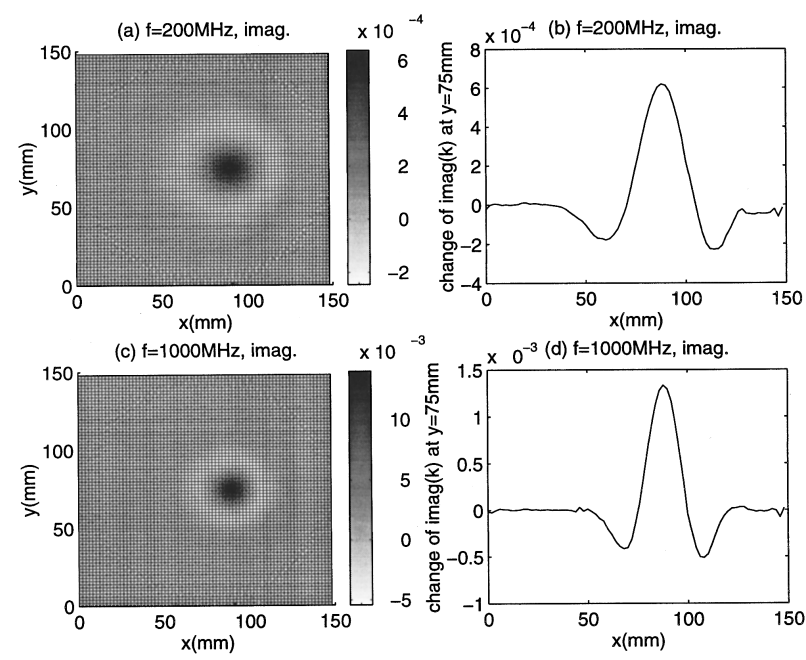

Fig. 42 -D test of $\delta \kappa_{i}(\mathbf{x})$ image reconstruction of $128 \times 128$ pixels. shrinkage effect implies that the MPDP carries more and more weight in determining $\phi(\mathbf{b}, \mathbf{a} ; \omega)$ and $\phi_{0}(\mathbf{b}, \mathbf{a} ; \omega)$ at the detector position at higher and higher modulation frequencies, and hence the WKB approximation becomes better and better as $\omega$ increases.

From Figures 3 and 4, the central position of the image object is nearly the same for different modulation frequencies. The reconstructed objects are larger than the actual objects. The figures also show that higher modulation frequencies give better images. Image reconstruction from phase information (the imaginary part) also gives a better resolution than reconstruction from amplitude data.

\subsection{IMAGE RECONSTRUCTION OF SIMULATED DATA FOR A FINITE MEDIUM}

In order to simulate the breast more closely, a medium in the shape of a square $(2 D) 60 \times 60 \mathrm{~mm}^{2}$ (see Figure 5) was simulated. The optical parameters for the background medium were chosen to correspond realistically to those of tissue matter in the breast ${ }^{21-23}: l_{a 0}=125 \mathrm{~mm} \quad\left(\mu_{a 0}=0.008 \mathrm{~mm}^{-1}\right)$, and $l_{t 0}=1.0 \mathrm{~mm} \quad\left(\mu_{s 0}^{\prime}=1.0 \mathrm{~mm}^{-1}\right)$. A cylindrical shape-absorbing object $5 \mathrm{~mm}$ in diameter was placed slightly off the center of the square $(35 \mathrm{~mm}$,

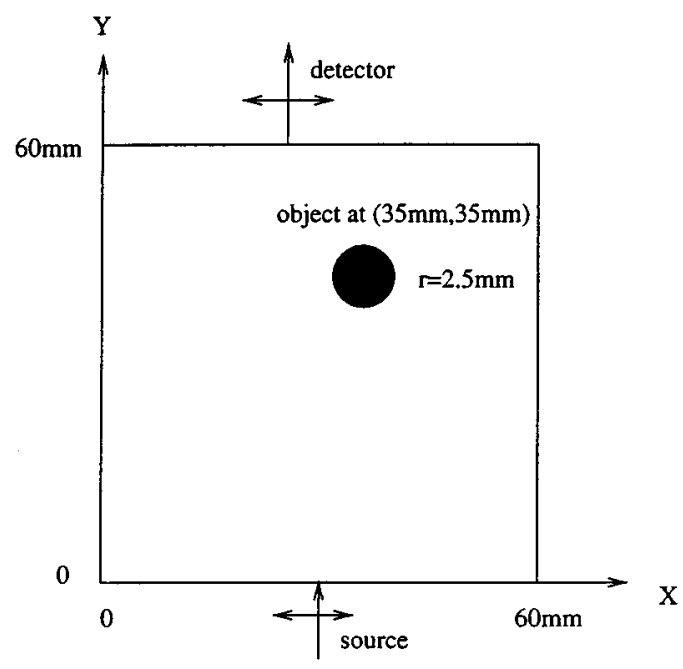

Fig. 5 A schematic of square geometry for simulated data for an absorbing boundary in a finite medium. 

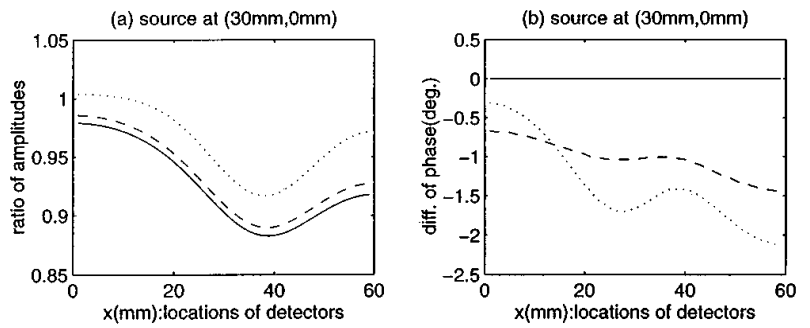

Fig. 6 Relationship of (a) the ratio (with object/without one) of the amplitudes and (b) the phase-shift difference (with object-without one) for various modulation frequencies. Solid, dashed, and dotted lines correspond to results for modulation frequencies of 0,200 , and $1000 \mathrm{MHz}$, respectively.

$35 \mathrm{~mm}$ ). The optical parameters of the hidden object were similar to those of a realistic tumor, with $\tilde{l}_{a 1}=45 \mathrm{~mm} \quad\left(\tilde{\mu}_{a 1}=0.024 \mathrm{~mm}^{-1}\right)$, and $\tilde{l}_{t 1}=1.0 \mathrm{~mm}$ $\left(\tilde{\mu}_{s 1}^{\prime}=1.0 \mathrm{~mm}^{-1}\right)$. The "measured" mock data were the photon fluxes, $J=-\left.D \nabla \phi\right|_{s}$, computed at the various detection positions by using numerical solutions of the diffusion equation. The solutions were computed with the absorbing boundary. ${ }^{6}$ The detectors and sources were arranged on opposite sides of the square and could be moved along opposite sides. The scan step size was $1 \mathrm{~mm}$, and the source-detector pairs were rotated $90 \mathrm{deg}$ once, so the total number of data points was $2 \times 59 \times 59$ $(=6962)$.

Figure 6 shows the relationship of the ratio (with object/without one) of the amplitudes and the phase-shift difference (with object-without one) for different modulation frequencies. The source was at the coordinate $(30 \mathrm{~mm}, 0 \mathrm{~mm})$ and the 59 detectors were uniformly placed at the coordinates of $y=60 \mathrm{~mm}$, and $x=1 \mathrm{~mm}, 2 \mathrm{~mm}, \ldots$, and $59 \mathrm{~mm}$. The changes in amplitude ratio and phase shift are similar to those discussed in Sec. 3.1.

Figure 7 shows images reconstructed from simulated data for various modulation frequencies by using the amplitude of the signals. Figures 7(a), $7(b)$, and 7(c) are gray-scale representations of the reconstructed images $\delta \kappa_{r}(\mathbf{x}, f)$ and correspond to $f=0,200$, and $1000 \mathrm{MHz}$, respectively. Figure 7(d) plots $\delta \kappa_{r}(\mathbf{x}, f)$ against spatial coordinates $x$ at $y$ $=35 \mathrm{~mm}$, i.e., the maximum slice plane of $\delta \kappa_{r}(\mathbf{x}, f)$. The central position of the reconstructed object is correct for all three modulation frequencies of 0,200 , and $1000 \mathrm{MHz}$. The maximum values of $\delta \kappa_{r}$ and half-widths of maximum of $\delta \kappa_{r}$ for different modulation frequencies are listed in Table 2.

These values show that the the reconstructed object is larger than the real one $(5 \mathrm{~mm})$; however, the size of the reconstruction becomes smaller when the modulation frequency is increased. The image quality (size and shape) is not as good as the reconstructed images for an infinite medium (see Figures 3 and 4). The dependence on modulation frequency is not as strong as in infinite media, but a higher frequency yields slightly better resolution. The rea-
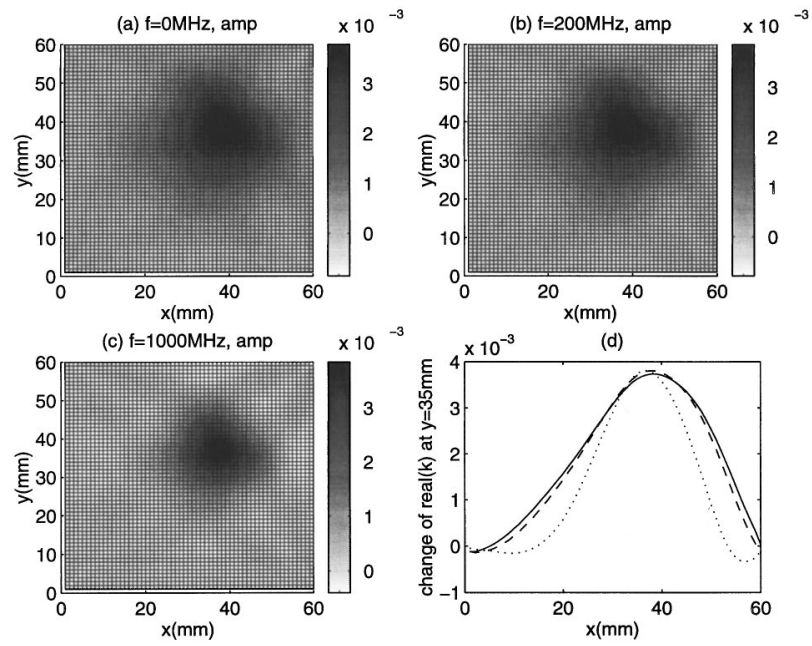

Fig. 7 2-D test of $\delta \kappa_{r}(\mathbf{x})$ image reconstruction of $60 \times 60$ pixels. In (d), the solid, dashed, and dotted lines correspond to modulation frequencies of 0,200 , and $1000 \mathrm{MHz}$, respectively.

son may be because a finite boundary and a limited number of projection directions are used (only two directions, so the shape of the image object is not a cylinder).

We have attempted to reconstruct images from phase information (imaginary parts of signals). Unfortunately, the image quality is not as good as images reconstructed from real amplitude due to the presence of the boundary.

\subsection{IMAGE RECONSTRUCTION FROM EXPERIMENTAL DATA}

Experiments were performed on an infinite Intralipid phantom medium at the zero modulation frequency. The medium was $40 \times 40 \times 30 \mathrm{~cm}^{3}$. Figure 8 shows a schematic of the experimental arrangement. Two laser diodes at wavelengths of $\lambda$ $=780 \mathrm{~nm}$ and $830 \mathrm{~nm}$ were used as sources. A mechanical chopper modulated the intensity of the laser beams at $350 \mathrm{~Hz}$. The laser beams were coupled into an optical fiber with diameter of $0.4 \mathrm{~mm}$ to deliver the light to the sample. The average power incident to the sample was about $2 \mathrm{~mW}$ for each wavelength. An optical fiber bundle with diameter of $5 \mathrm{~mm}$ was used to collect the scattered light. The output was split equally and coupled through two narrow band filters (780 and $830 \mathrm{~nm}$ ) to two photo-

Table 2 Effect of modulation frequency in finite media.

\begin{tabular}{rccc}
\hline$f(\mathrm{MHz})$ & $\left.\delta \kappa_{r}\right|_{\max }\left(\mathrm{mm}^{-1}\right)$ & $H W_{r}(\mathrm{~mm})$ & Object size $(\mathrm{mm})$ \\
\hline 0 & 0.0034 & 32 & 5 \\
200 & 0.0035 & 30 & 5 \\
1000 & 0.0035 & 22 & 5 \\
\hline
\end{tabular}




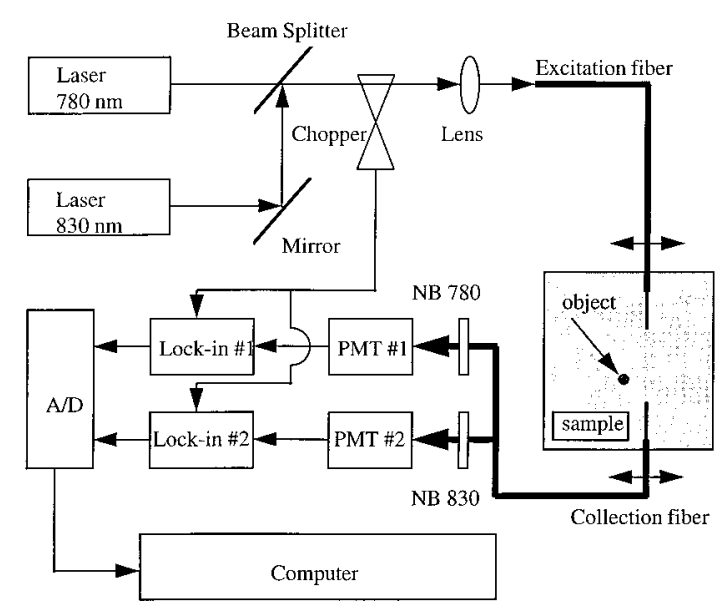

Fig. 8 A schematic diagram of experimental apparatus.

multipliers (PMT). Two PC board lock-in amplifiers (Ithaca 3981) detected and digitized the signal from the PMTs on a PC computer.

Figures 9(a) and 9(b) show the measured configuration of two phantom samples that were similar to the simulated example in Sec. 3.2. The optical parameters of the background media were about $l_{a 0}$ $=200 \mathrm{~mm}\left(\mu_{a 0}=0.005 / \mathrm{mm}\right.$, water $)$ and $l_{t 0}^{\prime}=5 \mathrm{~mm}$ $\left(\mu_{s 0}^{\prime}=0.2 / \mathrm{mm}\right)$. In order to test our algorithm, we assumed that the optical parameters of the background medium and object are unknown; therefore
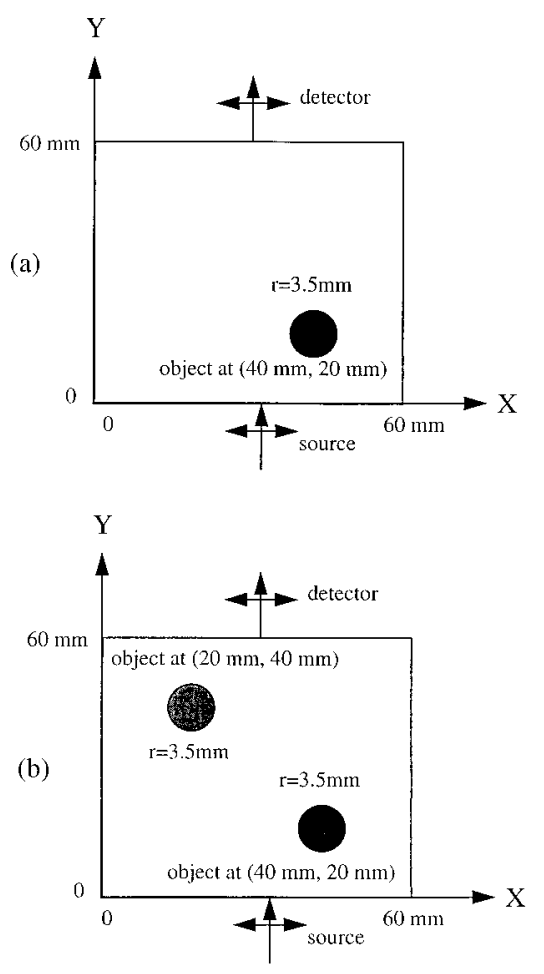

Fig. 9 A schematic of two phantom samples. (a) One cylindrical object filled with a stronger scattering and absorbing medium. (b) Two cylindrical objects with the same absorbing properties as their background.
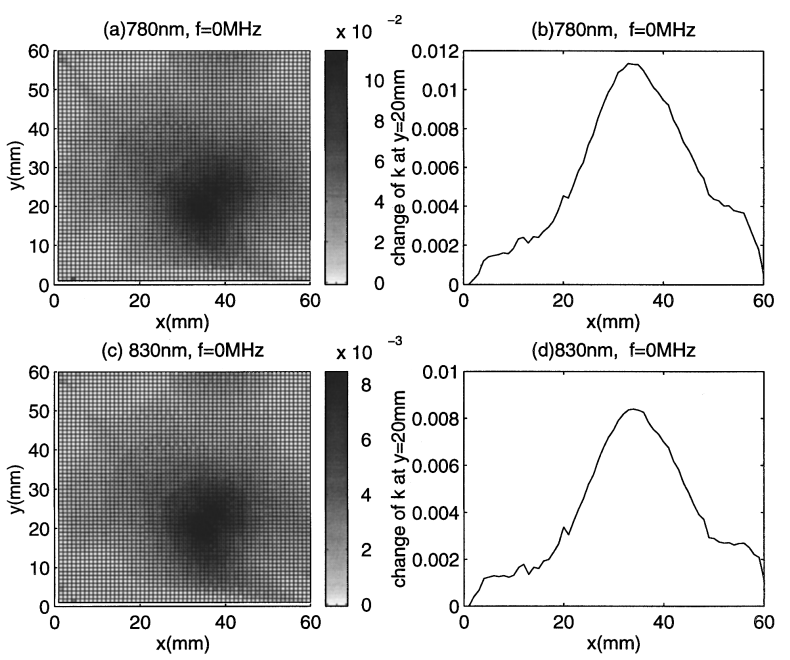

Fig. 10 2-D test of an image of $60 \times 60$ pixels reconstructed from the experimental data of an object [see Figure 9(a) and text].

Eqs. (7) and (8) must be employed again. The imaged area was a $60-\mathrm{mm}$ square in an infinite medium. The objects were cylinders with diameters of $7 \mathrm{~mm}$.

Similar to the measurement configuration in Sec. 3.2 , the source and the detector were attached to two opposite sides of the square sample region and moved along the two opposite sides. The source was moved in steps of $2 \mathrm{~mm}$, while the detector was moved in steps of $1 \mathrm{~mm}$. Therefore, $31 \times 59$ $=1829$ data points (dc intensity, $\omega=0$ ) were obtained for each projection direction. The sourcedetector pairs were rotated 90 deg once. The total data points were $2 \times 31 \times 59=3658$.

Two experiments were performed to demonstrate the potential of the algorithm. The first experimental square sample [see Figure 9(a)] has only one cylindrical object filled with a stronger scattering and absorbing medium. The object's medium was Intralipid solution with a small amount of naphthol green $\mathrm{B}$ dye. The optical parameters were about $l_{a}$ $=4.5 \mathrm{~mm} \quad\left(\mu_{a}=0.23 / \mathrm{mm}\right)$ at $780 \mathrm{~nm}$ and $l_{a}$ $=6.7 \mathrm{~mm}\left(\mu_{a}=0.15 / \mathrm{mm}\right)$ at $830 \mathrm{~nm} . l_{t}^{\prime}=1 \mathrm{~mm}$ $\left(\mu_{s}^{\prime}=1 / \mathrm{mm}\right)$ for 780 and $830 \mathrm{~nm}$. The coordinate of the center of the object was at $(40 \mathrm{~mm}, 20 \mathrm{~mm})$.

Figure 10 shows the reconstructed image of the object in Figure 9(a) in gray scale. Figures 10(a) and 10 (b) correspond to a source wavelength $\lambda$ $=780 \mathrm{~nm}$, and Figures 10(c) and 10(d) correspond to a source wavelength $\lambda=830 \mathrm{~nm}$. The shapes and sizes of the reconstructed images were about the same for the two source wavelengths. The value of $\delta \kappa_{r}$ at $780 \mathrm{~nm}$ was larger than the value at $830 \mathrm{~nm}$. This result is consistent with the fact that $\delta \kappa_{r}(780)$ $>\delta \kappa_{r}(830)$ for the object tested. The image shows that central position of the object is correct, but the shape of the image is not cylindrical and the size is much larger than the actual object. 

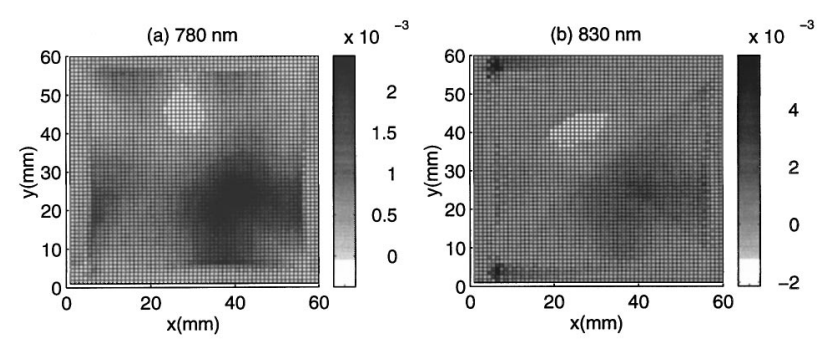

Fig. $112-D$ test of an image of $60 \times 60$ pixels reconstructed from the experimental data of two objects [see Figure $9(b)$ and text].

The second test sample is shown in Figure 9(b), where two cylindrical objects had the same absorbing properties as the background. One of the objects, centered at $(20 \mathrm{~mm}, 40 \mathrm{~mm})$, was nonscattering (water) and another object, centered at $(40 \mathrm{~mm}$, $20 \mathrm{~mm}$ ), was a higher scattering object, $l_{t}^{\prime}=1 \mathrm{~mm}$ $\left(\mu_{s}^{\prime}=1 / \mathrm{mm}\right)$.

Figure 11 shows the reconstructed result of two objects in Figure 9(b) in gray scale. Figure 11(a) corresponds to a source wavelength $\lambda=780 \mathrm{~nm}$; Figure 11(b) corresponds to a source wavelength $\lambda=830 \mathrm{~nm}$. A comparison of Figures 11(a) and 11(b) shows that a source wavelength $\lambda=780 \mathrm{~nm}$ is better than a source wave length $\lambda=830 \mathrm{~nm}$ for this sample. In Figure 11(a), one can resolve the two objects. The sizes of the images are different. This result is reasonable, because the two objects have the same absorbing properties as the background. The size of the nonscattering object is smaller and closer to the real size. The effect of diffusion blurring of the nonscattering object is much smaller than for the higher scattering object. It is also interesting to note that the value of $\delta \kappa$ for the nonscattering object is negative while it is positive for the strongly scattering object. This correctly indicates which object has a weaker attenuation factor. Figure 11(b) shows that at the $\lambda=830 \mathrm{~nm}$ the stronger scattering object was not clearly resolved, while the weaker object was better identified. The reason could be that at $\lambda=830 \mathrm{~nm}$ the scattering difference between object and background is smaller than that at $\lambda=780 \mathrm{~nm}$ for the strongly scattering object.

\section{DISCUSSION}

The results of image reconstruction using both simulated and experimental data with the WKB approximation algorithm show the following:

1 . Figures $3,4,6,7,10$, and 11 show that the central position(s) of hidden object(s) can be found. It is also possible to determine whether an object has a higher or lower photo density wave attenuation factor $\kappa$. However, the images are larger than the actual sizes of the objects in an infinite medium; the reconstructions using real parts are about 4.2, 4, and 3.2 times the size of the object for $f=0,200$, and $1000 \mathrm{MHz}$, respectively. The reconstructions using imaginary parts are about 2.4 and 1.4 times the size of the object for $f=200$ and $1000 \mathrm{MHz}$, respectively. For a finite medium, all reconstructions were at least four times larger than the actual objects.

These results show that the image was blurred by the diffusive nature of photons. Although the total amount of input data was about the same, the shapes of the reconstructed objects shown in Figures 3 and 4 are much closer to the real shape of a cylinder than the results shown in Figures 7, 10, and 11. The data used to reconstruct images in Figures 3 and 4 are full view angle data, while the data used for Figures 7, 10, and 11 are data with limited view angles. This result indicates that the experimental arrangement of geometry is important to this algorithm.

2. Tables 1 and 2 show that increasing the modulation frequency can improve image quality. However, the results show that for the modulation frequency $(200 \mathrm{MHz})$ commonly used, the improvement in the spatial resolution is limited. The spatial resolution can be significantly improved by using a much higher modulation frequency $(1000 \mathrm{MHz})$. This verifies that increasing modulation frequency leads to a shrinkage in the width of the "banana" region connecting the source and detector in which photon diffusion paths are concentrated. Too high a modulation frequency leads to a reduction in scattered light intensity and thus the signal-to-noise ratio. In the case discussed in Sec. 3.1, the intensity at $1000 \mathrm{MHz}$ is reduced more than 5 orders compared with the intensities at $\mathrm{CW}$ and $200 \mathrm{MHz}$.

3. The advantage of this algorithm is that the optical parameters need not be known. Figures 10 and 11 show that different source wavelengths produces different image results. Selecting a source wavelength for the absorption features of hidden objects should be considered for different samples.

4. The time to reconstruct an image of $N \times N$ $=128 \times 128$ pixels was about $5 \mathrm{~min}$ on the SUNSPARC 5. The memory needed is also small, proportional to a number of pixels $N$, while for the inverse matrix method, the computation time is about $N^{\log _{2} 7}$ and the memory size is $N^{2}$.

In summary, we have tested a novel diffusion tomography algorithm for imaging highly scattering media. Reconstruction results using both simulated data and experimental data show the capability of the WKB algorithm.

\section{Acknowledgment}

This work is supported in part by the New York State Science and Technology Foundation, NASAInstitutional Research Award Program, and Mediscience Technology Corp. The WKB image reconstruction algorithm was developed by Prof. S. C. Feng, Dr. H. L. Zhao, and one of us (F. Zeng) at the University of California at Los Angeles. We dedicate this paper to the memory of the late Prof. S. C. 
Feng. His insight in photon migration and diffusive photon imaging will be greatly missed.

\section{APPENDIX}

This appendix presents the deviation of forward solutions of diffusion equations [Eqs. (1) and (2)] in the path integration formula. This work was adopted from the work by S. C. Feng, F. Zeng, and H. L. Zhao at the University of California at Los Angeles. $^{13,14}$

The diffusion equation (1) can be described by path integration, following the original work of Feynman, ${ }^{15}$ i.e., if a photon has a mass $1 /(2 D)$ and moves in potential $-\mu_{a}(\mathbf{x})$, then we can use classical mechanics to describe the photon diffusion problem. In classical mechanics, the Lagrangian $L(\mathbf{x}, \dot{\mathbf{x}})$ of the diffusion equation (1) and classical action $S\{\mathbf{x}\}$ are, respectively

$$
L \equiv L(\mathbf{x}, \dot{\mathbf{x}})=\frac{1}{4 D c} \dot{\mathbf{x}}^{2}+\mu_{a}(\mathbf{x}) c,
$$

and

$$
S[\mathbf{x}(t)]=\int_{0}^{t} L d \tau=\int_{0}^{t}\left[\frac{1}{4 D c} \dot{\mathbf{x}}^{2}+\mu_{a}(\mathbf{x}) c\right] d \tau .
$$

The solution to Eq. (1) can be written as a path integration in a time domain as follows:

$$
\phi(\mathbf{b}, t ; \mathbf{a}, 0)=\int_{\mathbf{a}}^{\mathbf{b}} d \mathbf{x}(\tau) \exp \{-S[\mathbf{x}(\tau)]\},
$$

which describes diffusive photon propagation from source point $\mathbf{a}$ at time 0 to detector point $\mathbf{b}$ at time $t$. The integration $d \mathbf{x}(\tau)$ in Eq. (16) denotes a path integration over all the possible diffusion paths described by the trajectory $\mathbf{x}(\tau)$, with $0 \leqslant \tau \leqslant t$ being a running time variable.

Using the well-known stationary phase approximation, the lowest-order Green's function $\phi(\mathbf{b}, t ; \mathbf{a}, 0)$ is determined by minimizing the classical action $S[\mathbf{x}(\tau)]$, which determines the path $\mathbf{x}(\tau)$; this is a most probable diffusing path of the diffusion photon, with the starting point $(\mathbf{x}=\mathbf{a}$, at $\tau=0)$ and ending point $(\mathbf{x}=\mathbf{b}$, at $\tau=t)$.

The extremum property of $S[\mathbf{x}(\tau)]$ is expressed by $\delta S[\mathbf{x}(\tau)]=0$, which leads to the Euler-Lagrange equations for $\mathbf{x}(\tau)$ :

$$
\frac{d}{d t}\left(\frac{\partial L}{\partial \dot{x}}\right)-\frac{\partial L}{\partial x}=0
$$

Substituting Eq. (14) in Eq. (17), we can see that $\mathbf{x}(\tau)$ must satisfy:

$$
\frac{1}{2 D c} \ddot{\mathbf{x}}-\nabla \mu_{a}(\mathbf{x}) c=0 .
$$

An obvious necessary condition for $\mathbf{x}(\tau)$ to be a minimum of $S$ is that the quadratic form $\delta^{2} S$ be nonnegative. This requirement is valid for Lagrangians of Eq. (14). Expanding action $S[\mathbf{x}(\tau)]$ around $\overline{\mathbf{x}}(\tau)$, we have

$$
S[\mathbf{x}(\tau)]=S[\overline{\mathbf{x}}(\tau)]+\varepsilon \delta S+\frac{\varepsilon^{2}}{2 !} \delta^{2} S+\ldots
$$

Substituting Eq. (19) in Eq. (16), performing the integration up to the $\delta^{2} S$ (note: $\delta S=0$ ), after the tedious job of evaluating, one finds that the semiclassical WKB approximation for the diffusive Green's function ${ }^{15}$ is as follows:

$\phi(\mathbf{b}, t ; \mathbf{a}, 0)= \begin{cases}{\left[\left|\operatorname{det}\left(\frac{1}{2 \pi} \frac{\partial^{2} \bar{S}}{\partial \mathbf{b} \partial \mathbf{a}}\right)\right|\right]^{1 / 2} \exp (-\bar{S})} & t>0, \\ 0 & t<0,\end{cases}$

where $\bar{S} \equiv S\{\overline{\mathbf{x}}(\tau)\}$. The det is an $n$-dimensional determinant.

As a check for WKB approximation, solution of Eq. (18) for a homogeneous medium (note: $\nabla \mu_{a}(\mathbf{x})$ $=0)$ gives

$$
\overline{\mathbf{x}}(\tau)=\mathbf{a}+\frac{\tau}{t}(\mathbf{b}-\mathbf{a}),
$$

which indicates that the MPDP is a straight line for a homogeneous medium in a time domain.

After computing a classical action using Eq. (15), the time domain diffusion propagator of the homogeneous medium can be obtained from Eq. (20):

$$
\phi_{0}(\mathbf{b}, t ; \mathbf{a}, 0)=\left(\frac{1}{4 \pi D_{0} c t}\right)^{3 / 2} \exp \left(-\frac{(\mathbf{b}-\mathbf{a})^{2}}{4 D_{0} c t}-\mu_{a 0} c t\right),
$$

which is exactly the solution of the diffusion equation (1) for a homogeneous medium.

One can also formulate this WKB approximation in frequency domain. The photon fluence function $\phi(\mathbf{b}, \mathbf{a}, \omega)$ is obtained through Fourier transform as follows:

$$
\begin{aligned}
\phi(\mathbf{b}, \mathbf{a} ; \omega)= & \int_{-\infty}^{+\infty} d t \phi(\mathbf{b}, t ; \mathbf{a}, 0) \exp (i \omega t) \\
= & \int_{-\infty}^{+\infty} d t\left[\left|\operatorname{det}\left(\frac{1}{2 \pi} \frac{\partial^{2} \bar{S}}{\partial \mathbf{a} \partial \mathbf{b}}\right)\right|\right]^{1 / 2} \\
& \times \exp [-\bar{s}(\mathbf{b}, \mathbf{a}, t)+i \omega t] .
\end{aligned}
$$

Applying the stationary phase approximation from statistical mechanics, a special time scale $t_{0}$ can be identified to minimize $[\bar{S}(\mathbf{b}, \mathbf{a}, t,-i \omega t)]$ through: 


$$
\left.\frac{\partial \bar{S}(\mathbf{b}, \mathbf{a}, t)}{\partial t}\right|_{t_{0}}=i \omega
$$

For each special time scale $t_{0}$, a Gaussian approximation to the exponent of Eq. (23) at $t=t_{0}$ gives the leading term in $d$ space dimension:

$$
\begin{aligned}
\phi(\mathbf{b}, \mathbf{a} ; \omega)= & \left(\frac{1}{2 \pi}\right)^{d / 2}\left[\left|\operatorname{det} \partial^{2} \bar{S}\left(\mathbf{b}, \mathbf{a} ; t_{0}\right) / \partial \mathbf{b} \partial \mathbf{a}\right|\right]^{1 / 2} \\
& \times \exp \left[-\bar{S}+i \omega t_{0}\right] \int_{-\infty}^{\infty} d t \\
& \times \exp \left\{-\left[\partial^{2} \bar{S}\left(\mathbf{b}, \mathbf{a} ; t_{0}\right) / \partial t^{2}\right]\left(t-t_{0}\right)^{2} / 2\right\}
\end{aligned}
$$

In Eq. (25), part of the integral is:

$$
\begin{gathered}
\int_{-\infty}^{\infty} d t \exp \left\{-\left[\partial^{2} \bar{S}\left(\mathbf{b}, \mathbf{a} ; t_{0}\right) / \partial t^{2}\right]\left(t-t_{0}\right)^{2} / 2\right\} \\
=\left[\frac{2 \pi}{\partial^{2} \bar{S}\left(\mathbf{b}, \mathbf{a} ; t_{0}\right) / \partial t_{0}^{2}}\right]^{1 / 2} .
\end{gathered}
$$

Using the standard Legendre transformation:

$$
W(\mathbf{b}, \mathbf{a} ; \omega)=\bar{S}\left(\mathbf{b}, \mathbf{a} ; t_{0}\right)-i \omega t_{0},
$$

Eq. (25) can be rewritten as:

$$
\phi(\mathbf{b}, \mathbf{a} ; \omega)=\left(\frac{1}{2 \pi}\right)^{d-1 / 2} \sqrt{\tilde{D}} \exp [-W(\mathbf{b}, \mathbf{a} ; \omega)]
$$

where $\tilde{D}$ is

$$
\begin{aligned}
\tilde{D} & =\operatorname{det}\left(\frac{\partial^{2} \bar{S}\left(\mathbf{b}, \mathbf{a} ; t_{0}\right)}{\partial \mathbf{b} \partial \mathbf{a}}\right) / \frac{\partial^{2} \bar{S}\left(\mathbf{b}, \mathbf{a} ; t_{0}\right)}{\partial t_{0}^{2}} \\
& =\operatorname{det}\left[\begin{array}{cc}
\frac{\partial^{2} W}{\partial \mathbf{b} \partial \mathbf{a}} & i \frac{\partial^{2} W}{\partial \mathbf{b} \partial \omega} \\
i \frac{\partial^{2} W}{\partial \mathbf{a} \partial \omega} & -\frac{\partial^{2} W}{\partial \omega^{2}}
\end{array}\right] .
\end{aligned}
$$

From the Hamilton-Jacobi equation for action $S$, we have

$$
D\left(\frac{\partial W}{\partial \overline{\mathbf{x}}}\right)^{2}-\mu_{a}(\overline{\mathbf{x}})=-i \omega
$$

Thus, $W(\mathbf{b}, \mathbf{a} ; \omega)$ corresponds to the path integration as follows:

$$
W(\mathbf{b}, \mathbf{a} ; \omega)=\int_{\mathbf{a}}^{\mathbf{b}} \kappa(\mathbf{x}, \omega) d \mathbf{l}
$$

where

$$
\kappa(\mathbf{x}, \omega)=\left[\mu_{a} / D-i \omega / D c\right]^{1 / 2}
$$

$$
\begin{aligned}
= & \frac{1}{\sqrt{2 D}}\left\{\left[\sqrt{\mu_{a}^{2}+(\omega / c)^{2}}+\mu_{a}\right]^{1 / 2}\right. \\
& \left.-i\left[\sqrt{\mu_{a}^{2}+(\omega / c)^{2}}-\mu_{a}\right]^{1 / 2}\right\} .
\end{aligned}
$$

For homogeneous media, from Eq. (31), $W(\mathbf{b}, \mathbf{a} ; \boldsymbol{\omega})=\kappa_{0}|\mathbf{b}-\mathbf{a}|$, and from Eq. (29), $\tilde{D}$ $=\left[1 /\left(2 D_{0}|\mathbf{b}-\mathbf{a}|\right)^{2}\right]$; thus, for a three-space dimension $d=3$, Eq. (28) becomes

$$
\phi_{0}(\mathbf{b}, \mathbf{a}, \omega)=\frac{1}{4 \pi D_{0} c r} \exp \left(-\kappa_{0} r\right),
$$

where $r=|\mathbf{b}-\mathbf{a}|$. Equation (33) is the solution of the diffusion equation (1) for homogeneous media in a frequency domain.

For inhomogeneous media, the MPDP in the frequency domain can be obtained using the variational principle ${ }^{24}$

$$
\delta W(\mathbf{b}, \mathbf{a}, \omega)=\delta \int_{\mathbf{a}}^{\mathbf{b}} \kappa(\mathbf{x}, \omega) d l=0 .
$$

Since $\kappa(\mathbf{x}, \omega)$ is a complex, this equation is true for both the real and imaginary parts of $\kappa(\mathbf{x}, \omega)$. Explicit expression of the MPDP can be obtained using differential geometry. ${ }^{24}$ If we denote $\mathbf{t}, \mathbf{n}$ as the unit tangential and principal normal vectors of the MPDP, and let $R$ be the local radius of curvature of the MPDP, these quantities can be obtained from the trajectory function of the MPDP $\mathbf{x}(l)$ by the relations

$$
\mathbf{t}=\frac{d \mathbf{x}}{d l}, \quad \frac{\mathbf{n}}{R}=\frac{d^{2} \mathbf{x}}{d l^{2}} .
$$

The MPDP is then determined from

$$
\begin{aligned}
\nabla \mu_{a}-\left(\mathbf{t} \cdot \nabla \mu_{a}\right) \mathbf{t} & =\left(\nabla \mu_{a}\right)_{n} \mathbf{n} \\
& =2\left[\left(\mu_{a} / D\right)^{2}+(\omega / D c)^{2} \frac{\mathbf{n}}{R}\right]^{1 / 2} .
\end{aligned}
$$

An interesting quantitative feature of Eq. (36) is that for a given spatial distribution of $\mu_{a}(\mathbf{x})$, when modulation frequencies are increased, the radius of curvature $R$ is also increased [note: the spatial distribution of $\mu_{a}(\mathbf{x})$ is given]. This is why the MPDP becomes straighter when the modulation frequencies are increased. This point can be seen in Sec. 3 . Analytic solutions of Eq. (36) for an arbitrary potential $\mu_{a}(\mathbf{x})$ are in general difficult. However, it is possible to numerically "trace out" an MPDP in the frequency domain through Eq. (36). Once the MPDP path is obtained, one can numerically integrate $\kappa(\mathbf{x}, \omega)$ along the path, and obtain the photon fluence rate in the $\mathrm{WKB}$ approximation at the endpoint $\mathbf{x}=\mathbf{b}$ through Eq. (2). Obviously, if $\nabla \mu_{a} \rightarrow 0$, then $R \rightarrow \infty$; this indicates that for homogeneous media, MPDP is a straight line for any modulation frequency. 


\section{REFERENCES}

1. Shechao Feng, Fanan Zeng, and Britton Chance, "Perturbation theory of photon migration in the presence of a single defect," in Proceedings on Advances in Optical Imaging and Photon Migration, R. R. Alfano, Ed., Vol. 21, pp. 217-228, Optical Society of America, Washington, DC (1994).

2. R. R. Alfano, X. Liang, L. Wang, and P. P. Ho, "Timeresolved imaging of translucent droplets in highly scattering turbid media," Science 264; 1913-1915 (1994).

3. S. R. Arridge, "The forward and inverse problems in time resolved infrared imaging," in Medical Optical Tomography: Functional Imaging and Monitoring, G. Muller et al. ed., SPIE Vol. IS11, pp. 35-64 (1993).

4. M. A. O'Leary, D. A. Boas, B. Chance, and A. G. Yodh, "Images of inhomogeneous turbid media using diffuse photon density waves," in Proceedings on Advances in Optical Imaging and Photon Migration, R. R. Alfano, Ed., Vol. 21, pp. 106-115 Optical Society of America, Washington, DC (1994).

5. B. Chance, "Time resolved spectroscopy and imaging," Proc. SPIE 2389, 122-139 (1995).

6. W. Cai, B. B. Das, Feng Liu, M. Zevallos, M. Lax, and R. R. Alfano, "Time-resolved optical diffusion tomographic image reconstruction in highly scattering turbid media," Proc. Natl. Acad. USA 93, pp. 13561-13564 (1996).

7. J. C. Schotland, J. C. Haselgrove, and J. S. Leigh, "Photon hitting density," Appl. Opt. 32(4), 448-453 (1993).

8. J. C. Schotland, "Inversion formulas for diffusion imaging," Proc. SPIE 2389, 304-310 (1995).

9. H. L. Graber, J. Chang, J. Lubowsky, R. Aronson, and R. L. Barbour, "Near-infrared absorption imaging of dense scattering media by steady-state diffusion tomography," Proc. SPIE 1888, 372 (1993).

10. S. A. Walker, A. E. Cerussi, and E. Gratton, "Back-projection image reconstruction using photon density waves in tissues," Proc. SPIE 2389, 350-357 (1995).

11. H. B. Jing, K. D. Paulsen, U. L. Osterberg, and B. W. Pogue, "Optical image reconstruction using frequency-domain data - simulations and experiments," J. Opt. Soc. Am. A13, 253266 (1996).

12. B. W. Pogue, M. S. Patterson, H. B. Jiang, and K. D. Paulsen, "Initial assessment of a simple system for frequency domain diffuse optical tomography," Phys. Med. Biol. 40, 1709-1729 (1995).

13. S. C. Feng, H.-L. Zhao, and F.-A. Zeng, "New algorithm for near-infrared diffuse photon imaging," Proc. SPIE 2389, 113-119 (1995).

14. S. Feng, F. Zeng, and H. Zhao, "A methodology and apparatus for diffuse photon imaging," U.S. Pat. Appl. (1995) (UCLA/p353-D, LA95-046-01).

15. L. S. Schulman, Techniques and Applications of Path Integration, Wiley, New York (1981).

16. A. C. Kak and M. Slaney, Principles of Computerized Tomographic Imaging, IEEE Press (1988).

17. S. Jacques, A. Gutsche, J. Schwartz, Lihong Wang, and F. Tittel, "Video reflectometry to specify optical properties of tissue in vivo," SPIE Vol. IS11, Editors G. Muller, B. Chance, R. Alfano, et al., pp. 211-226 (1995).

18. H. J. C. M. Sterenborg, M. J. C. van Gemert, W. Kamphort, and J. G. Wolbers, "The spectral dependence of the optical properties of human brain," Lasers Med. Sci. 4, 221-227 (1989).

19. P. van der Zee, M. Essenpreis, and D. T. Delpy, "Optical properties of brain tissue," Proc. SPIE 1888, 454-465 (1993).

20. R. Splinter, W. F. Cheong, M. J. C. van Germert, and A. J. Welch, "In vitro optical properties of human and canine brain and urinary bladder tissue at $633 \mathrm{~nm}$," Lasers Surg. Med. 9, 37-41 (1989).

21. B. Barbieri, F. De Picoli, M. J. vande Ven, and E. Gratton, "What determines the uncertainty of phase and modulation measurements in frequency domain fluorometry?" Proc. SPIE 1204, 158-170 (1990).

22. K. A. Kang, B. Chance, S. Zhao, S. Srinivasan, E. Patterson, and R. Troupin, "Breast tumor characterization using nearinfra-red spectroscopy," Proc. SPIE 1888, 487-499 (1993).

23. Y. Yamashita and M. Kaneko, "Visible and infrared diaphanoscopy for medical diagnosis," in Medical Optical Tomography: Functional Imaging and Monitoring, SPIE Vol. IS11, pp. 283-316 (1993).

24. L. D. Landau and E. M. Lifshitz, "VII. The Canonical Equations, §44" in Mechanics, Course of Theoretical Physics, 3rd ed., Vol. 1, pp. 140-143, Pergamon Press, New York (1976). 